\title{
An Extra-Solar Planet in a Double Stellar System: The Modelling of Insufficient Orbital Elements
}

\author{
Eva Plávalová ${ }^{1}$, Nina A. Solovaya ${ }^{2,3}$ and Eduard M. Pittich ${ }^{2}$ \\ ${ }^{1}$ Dept. of Astronomy, Earth's Physics, and Meteorology, Comenius University, Bratislava, \\ Slovak Republic, email: plavala@slovanet.sk \\ ${ }^{2}$ Astronomical Institute, Slovak Academy of Sciences, Bratislava, Slovak Republic \\ email: astrosol@savba.sk, pittich@savba.sk \\ ${ }^{3}$ Sternberg Astronomical Institute, Moscow State University, Moscow, Russia \\ email: solov@sai.msu.ru
}

\begin{abstract}
The modelling of the insufficient orbital elements of extra-solar planets (EPs) revolving around one component in a binary star system is investigated in the present paper. This problem is considered in the frame of the three-body problem using the analytical theory of Orlov \& Solovaya (1998). In the general case, the motion is defined by the masses of the components and by the six pairs of the initial values of the Keplerian elements. For EPs, it is not possible to obtain the complete set of elements for the orbit, in particular, the ascending node and the angle of the inclination. So, it is possible the two different variants of orbital evolution of EPs depend on the initial conditions. In one case, the orbit is unstable. Using the stability conditions of Solovaya \& Pittich (2004), which are presented by the angle of the mutual inclination of the orbits between the EP and distant star, we varied unknown angular elements and defined the regions with possible values of the elements for which the motion of EP stays stable. We applied these calculations to the particular specific EPs: HD19994b, HD196885Ab and HD222404b.
\end{abstract}

Keywords. three body problem, orbital stability of extra-solar planets, HD19994b, HD196885Ab and HD222404b

\section{Application of the theory}

We have investigated the motions of EPs for which the Keplerian orbital elements are incomplete. The possible conditions for dynamical stability are presented by the orbital parameters: the mutual inclination and argument of perigee of the EP. For dynamical stability, the eccentricity of the EP orbit remains less than 1 . The mutual inclination of the orbits changes within small limits and there is no close approach of the EP to the parent star.

\section{Calculations and results}

We do not know either the inclination of the orbit or the ascending node for EPs. We changed these unknown angular values in $1^{\circ}$ steps, calculated and determined regions where the motion is stable. They are situated in two stable regions with an identical shape. For the exclusion of the trigonometric drift region, we calculated stable values for the inclination of the EP which is orbiting on a circular orbit. The calculated values for the inclination of the EP for a circular orbit have the same values as for one of 
Table 1. Orbital elements for EPs and distant stars.

\begin{tabular}{|c|c|c|c|c|c|c|c|c|}
\hline Name & $\mathrm{C}$ & $\begin{array}{c}\text { Mass } \\
\left(M_{J u p}\right)\end{array}$ & $\begin{array}{c}\text { Mass } \\
\left(M_{S u n}\right)\end{array}$ & $(\stackrel{\mathrm{a}}{\mathrm{A} \mathrm{U}})$ & e & $\left(\begin{array}{l}\omega \\
\left({ }^{\circ}\right.\end{array}\right)$ & $\begin{array}{l}\Omega \\
\left({ }^{\circ}\right)\end{array}$ & $\left(\begin{array}{l}\mathrm{i} \\
\left({ }^{\circ}\right)\end{array}\right.$ \\
\hline HD 19994 & $\begin{array}{l}\mathrm{A} \\
\mathrm{B} \\
\mathrm{b}\end{array}$ & $1.68^{a}$ & $\begin{array}{l}1.34^{a} \\
0.022\end{array}$ & $\begin{array}{l}75.76^{b} \\
1.42^{a}\end{array}$ & $\begin{array}{c}0.26^{b} \\
0.3 \pm 0.04^{a}\end{array}$ & $\begin{array}{c}247.7^{b} \\
41 \pm 8^{a}\end{array}$ & $\begin{array}{c}84.13^{b} \\
67.5 \pm 41.5^{e}\end{array}$ & $\begin{array}{c}114.1^{b} \\
66.5 \pm 37.5^{e}\end{array}$ \\
\hline HD 196885 & $\begin{array}{l}\mathrm{A} \\
\mathrm{B} \\
\mathrm{b}\end{array}$ & $2.98 \pm 0.05^{a}$ & $\begin{array}{c}1.33^{a} \\
0.45 \pm 0.01^{c}\end{array}$ & $\begin{array}{c}21.00 \pm 0.86^{c} \\
2.6 \pm 0.1^{a}\end{array}$ & $\begin{array}{r}0.409 \pm 0.038^{c} \\
0.48 \pm 0.02^{a}\end{array}$ & $\begin{array}{c}227.6 \pm 23.4^{c} \\
93.2 \pm 3^{a}\end{array}$ & $\begin{array}{c}79.8 \pm 0.1^{c} \\
47.5 \pm 43.5^{e}\end{array}$ & $\begin{array}{c}116.8 \pm 0.7^{c} \\
64 \pm 31^{e}\end{array}$ \\
\hline H D 222404 & $\begin{array}{l}\mathrm{A} \\
\mathrm{B} \\
\mathrm{b}\end{array}$ & $1.85 \pm 0.06^{a}$ & $\begin{array}{c}1.4 \pm 0.12^{a} \\
0.362 \pm 0.022^{d}\end{array}$ & $\begin{array}{c}19.02 \pm 0.64^{d} \\
2.05 \pm 0.06^{a}\end{array}$ & $\begin{aligned} 0.4085 & \pm 0.0065^{d} \\
0.049 & \pm 0.034^{a}\end{aligned}$ & $\begin{array}{c}160.96 \pm 0.40^{d} \\
94.6 \pm 34.6^{a}\end{array}$ & $\begin{aligned} 13.0 & \pm 2.4^{d} \\
340.5 & \pm 45.5^{e}\end{aligned}$ & $\begin{array}{c}118.1 \pm 2.4^{d} \\
5.7^{+15.1 a^{a}} \\
\operatorname{or} 62 \pm 38^{e}\end{array}$ \\
\hline
\end{tabular}

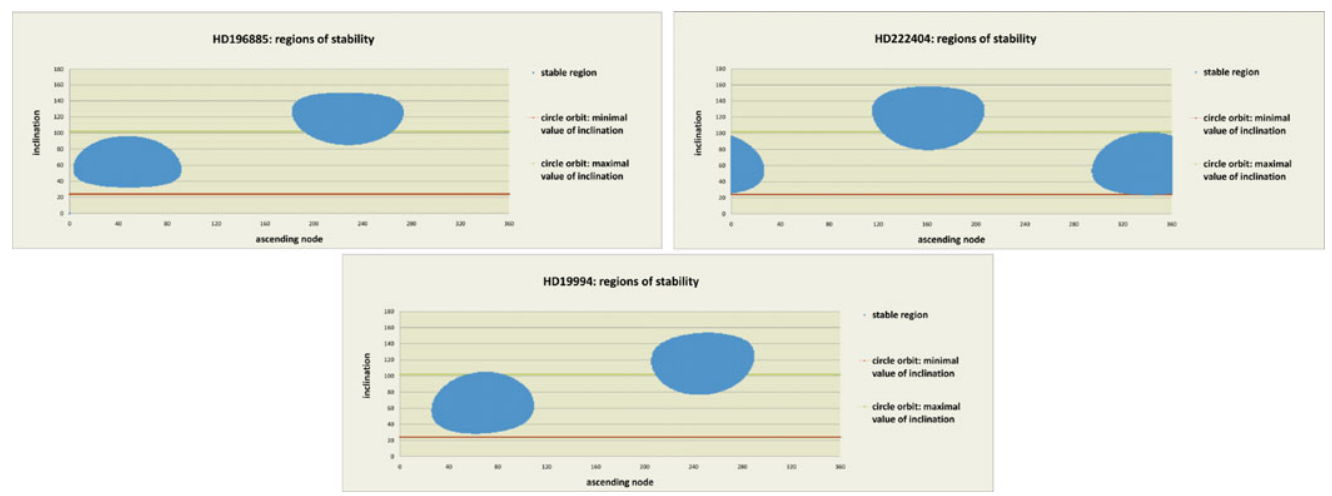

Figure 1. Relation diagram between inclination and ascending node for: HD196885Ab, HD222404b and HD19994b.

the high eccentricity orbits for stable regions (see Fig. 1). This stable region we could consider as a region with possible values for the insufficient Keplerian elements. As an example to illustrate the theory, we took three binary stellar systems with hosting EPs; HD196885Ab, HD222404b and HD19994b. Their known orbital elements for EPs and distant stars are in Table 1. The two possible stable regions are shown on Fig. 1.

We derived the following possible values for the insufficient Keplerian elements: the value of the inclination is $47.5^{\circ} \pm 43.5^{\circ}$ and for the node $64^{\circ} \pm 31^{\circ}$ for EP HD196885Ab, $66.5^{\circ} \pm 37.5^{\circ}$ and $67,5^{\circ} \pm 41.5^{\circ}$ for the EP HD19994Ab, and $62^{\circ} \pm 38^{\circ}$ and $340.5^{\circ} \pm 45.5^{\circ}$ for the EP HD222404b. The published value of the inclination for HD222404b by Schneider (2011) is quite different, $5.7_{-1.9^{\circ}}^{\circ}$.

\section{References}

Hartkopf, W. I. \& Mason, B. D. 2001, Sixth Catalog of Orbits of Visual Binary Stars Chauvin,G., Beust, H., Lagrange, A.-M., \& Eggenberger, A. 2011, A\&A, 528, A8

Orlov, A.A. \& Solovaya, N.A. 1998, in The Few Body Problem, ed. M.J. Valtonen, (Kluwer Acad. Publish., Dordrecht), 243

Schneider, J. 2011, Extra-solar Planets Catalogue, http://exoplanet.eu

Solovaya, N.A. \& Pittich, E.M. 2004, Contrib. Astron. Obs. Skalnaté Pleso, 34, 105

Torres, G. 2008, arXiv:astro-ph/0609638v1 